\title{
Nématodes parasites, nouveaux ou intéressants,
}

\section{du Vénézuéla}

\author{
Par Carlos DIAZ-UNGRIA
}

Une collection de Nématodes que nous avons récoltée au Vénézuela chez des animaux domestiques et sauvages, a été étudiée au cours d'un stage au laboratoire de Zoologie (Vers) du Muséum, sous la direction du Professeur A. Chabaud, à qui nous exprimons notre vive gratitude.

Les espèces apportant des éléments nouveaux pour la systématique des Nématodes sont étudiées ci-dessous. Les types sont déposés au Muséum National d'Histoire Naturelle à Paris.

I. - TRIODONTOPHORUS NIPPONICUS Yamaguti 1943 (= T. hsiungi K'ung 1958)

Nos spécimens récoltés chez des chevaux domestiques et des ânes de Maracay, ne diffèrent de la description de Yamaguti que par des éléments mineurs : le nombre des éléments de la coronule varie de 57 à 69 ; les spicules sont longs de 800 à $850 \mu$; la distance vulve-pointe caudale est de 725 à $950 \mu$.

La description de $T$. hsungi K'ung 1958 ne présente aucun élément différentiel et nous proposons donc la mise en synonymie de cette espèce avec nipponicus.

L'espèce décrite en Corée chez le cheval n'est donc pas localisée à cette région. Elle a été retrouvée une première fois en Chine par K'ung et une seconde fois au Vénézuela par nous-même. L'âne est un hôte nouveau.

$$
\text { II. - LONGISTRIATA DOLLFUSI n. sp. (fig. 3) }
$$

Les spécimens étudiés proviennent de l'intestin grêle de souris grises Mus musculus L., vivant en forêt, à Tiara (Aragua. Vénézuela).

\section{Description :}

Mâle : Corps long de 3,53 mm, large au maximum de $140 \mu$; cuticule striée transversalement. Vésicule cuticulaire céphalique longue de 65-70 $\mu$, large de 33-35 $\mu$. Quatre

* Extraits d'un travail présenté pour l'obtention du Diplôme de l'Ecole pratique des HautesEtudes, le 23 mars 1963.

Travail réalisé grâce à une subvention du Gouvernement vénézuélien et une bonrse d'étude du Gouvernement français.

Ann. de Parasitologie humaine et comparée (Paris), t. 38, 1963, $\mathrm{n}^{\circ} 6$ 
papilles céphaliques. Esophage claviforme long de $310 \mu$, large au maximum de $25 \mu$. Pore excréteur à $250 \mu$ de l'apex. Diérides et anneau nerveux non repérés. Bourse caudale avec deux lobes latéraux à peu près symétriques et lobe dorsal non individualisé, avec une profonde incision médiane qui arrive presque à la base des deux branches de
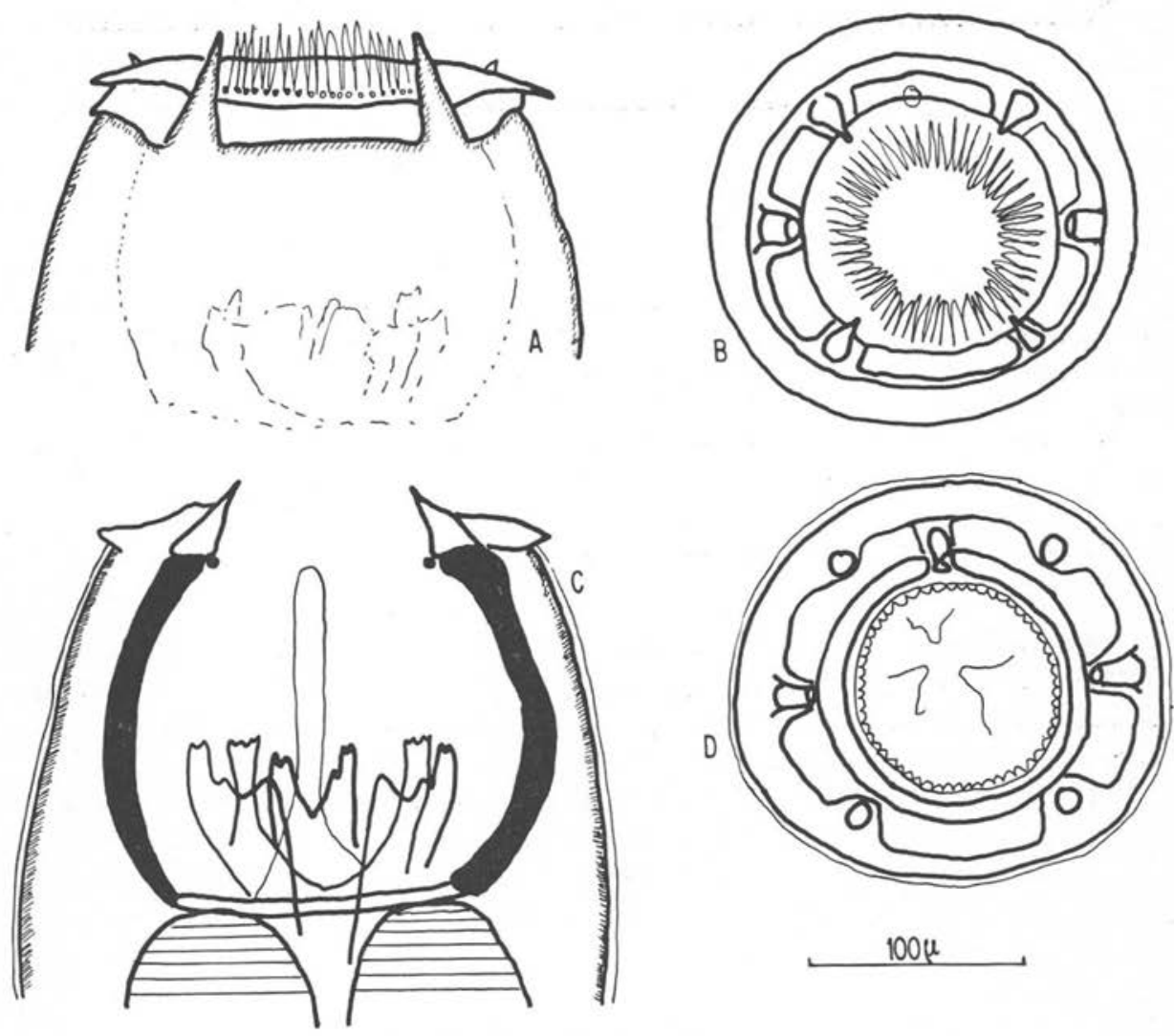

Triodontophorus nipponicus :

FIg. 1. - Extrémité céphalique. - A :vue ventrale superficielle. - B : vue frontale superficielle. $\mathrm{C}$ :vue ventrale profonde. - D :Vue frontale profonde.

la côte dorsale. La bourse caudale mesure 430-500 $\mu$ de large et 180-220 $\mu$ de long. La disposition des côtes est figurée en A et B. L'hypertrophie de l'antéro-latérale est remarquable. Spicules subégaux, long de 625-660 $\mu$, gubernaculum petit, en forme d'anneau ouvert en avant. Cône génital proéminent, finissant en deux mammelons.

Femelle: Longueur du corps très variable, de 2,84 à $6,56 \mathrm{~mm}$. Vésicule céphalique longue de 53-75 $\mu$, large de 28 à $38 \mu$. Esophage long de 300-360 $\mu$, large de 30 à $35 \mu$. 
Diérides et anneau nerveux non repérés. Pore excréteur à 180-190 $\mu$ de l'apex. Queue longue de $50-80 \mu$; vulve à $200-215 \mu$ de la pointe caudale. Entre la vulve et l'anus, la cuticule constitue une vaste dilatation circulaire en forme de prépuce. Ovéjecteur très court et flexueux suivi d'un utérus empli d'œufs. Ceux-ci mesurent 50-60 $\mu \times 29-35 \mu$.

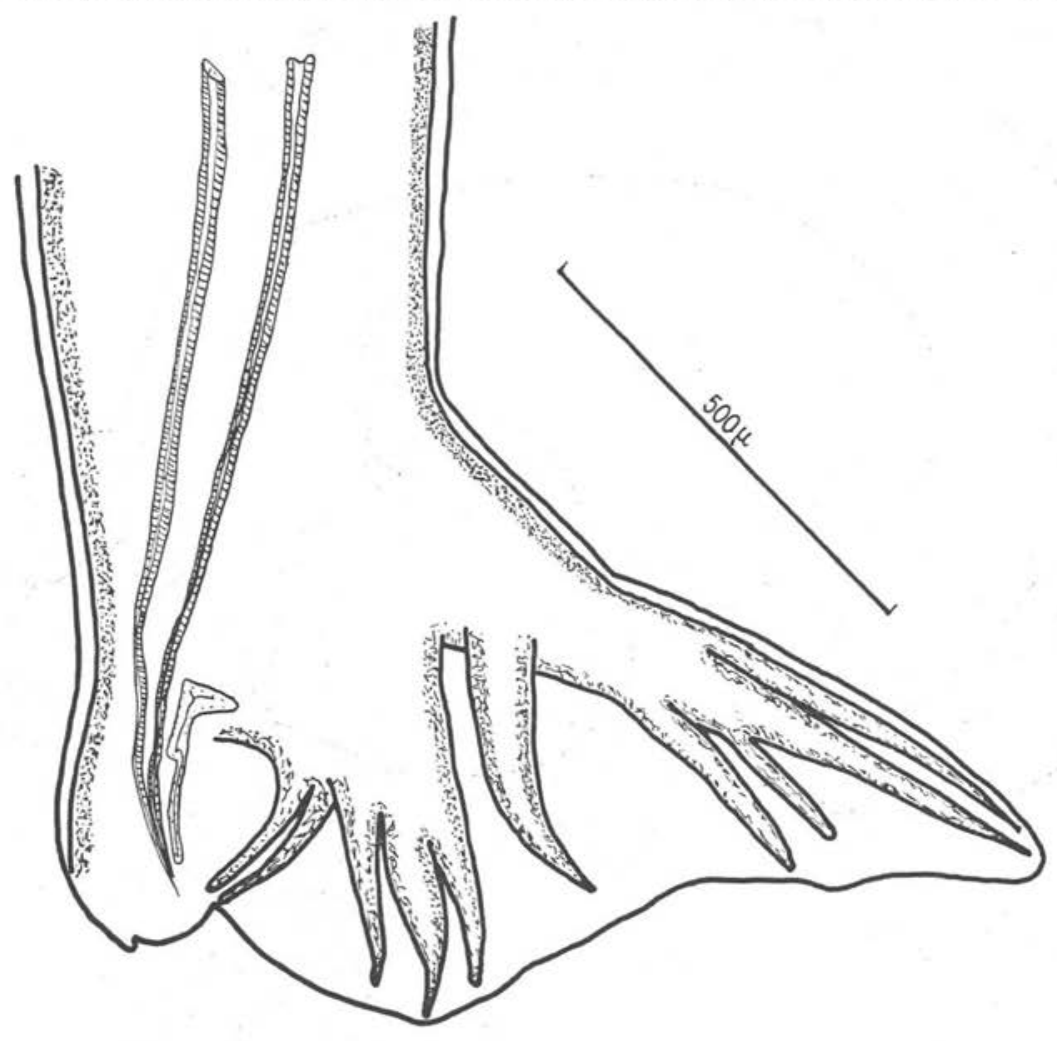

Triodontophorus nipponicus:

Fig. 2. - Extrémité postérieure du mâle: vue latérale

\section{Discussion :}

Notre espèce appartient au genre Longistriata Schulz 1926 sensu Chabaud 1959, mais possède des caractères très particuliers.

Trois espèces ont également une extrémité caudale femelle dilatée en forme de prépuce : L. maldonadoi Artigas et Pacheco 1953, L. dubia, (Travassos 1921) et L. convoluta Caballero et Cerecero 1942 ; cette dilatation est, cependant, mieux marquée chez notre espèce. L'élément le plus caractéristique de la forme décrite plus haut réside dans l'hypertrophie considérable de la côte antéro-latérale, qui n'a été signalée chez aucune autre espèce. Nous pensons donc que celle-ci est nouvelle et nous proposons le nom de 
Longistriata dollfusi $\mathrm{n}$. sp. en respectueux hommage à $\mathrm{M}$. Robert $\mathrm{Ph}$. Dollfus, Directeur Honoraire à l'Ecole Pratique des Hautes Etudes.

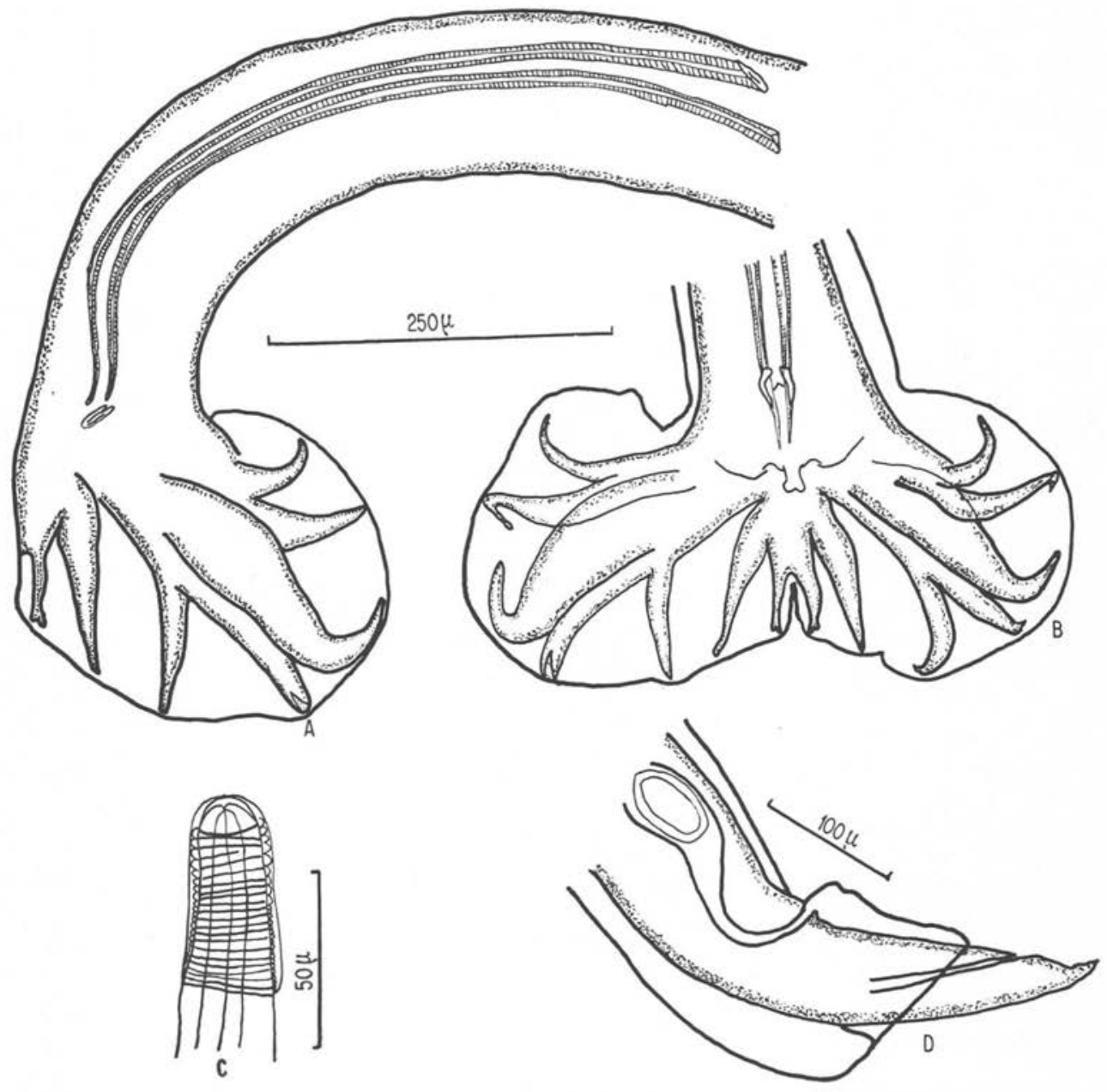

Longistriata dollfusi :

Fig. 3. - A: extrémité caudale du mâle, vue latérale. - B: extrémité caudale du mâle, vue ventrale. - C : extrémité antérieure, vue latérale. - D : extrémité caudale de la femelle, vue latérale.

III. - MARACAYA GRACIAI n. g., n. sp.

(fig. 4-5)

Nous avons récolté l'espèce dans l'intestin du Lézard Amphisbaene alba à Maracay. 


\section{Description :}

Tête avec trois grandes lèvres hautes d'environ $75 \mu$, une dorsale légèrement plus haute avec deux papilles, et, deux latéro-ventrales avec chacune une papille et une amphide. Le bord des lèvres est chitinoïde. La vue apicale profonde montre une pièce
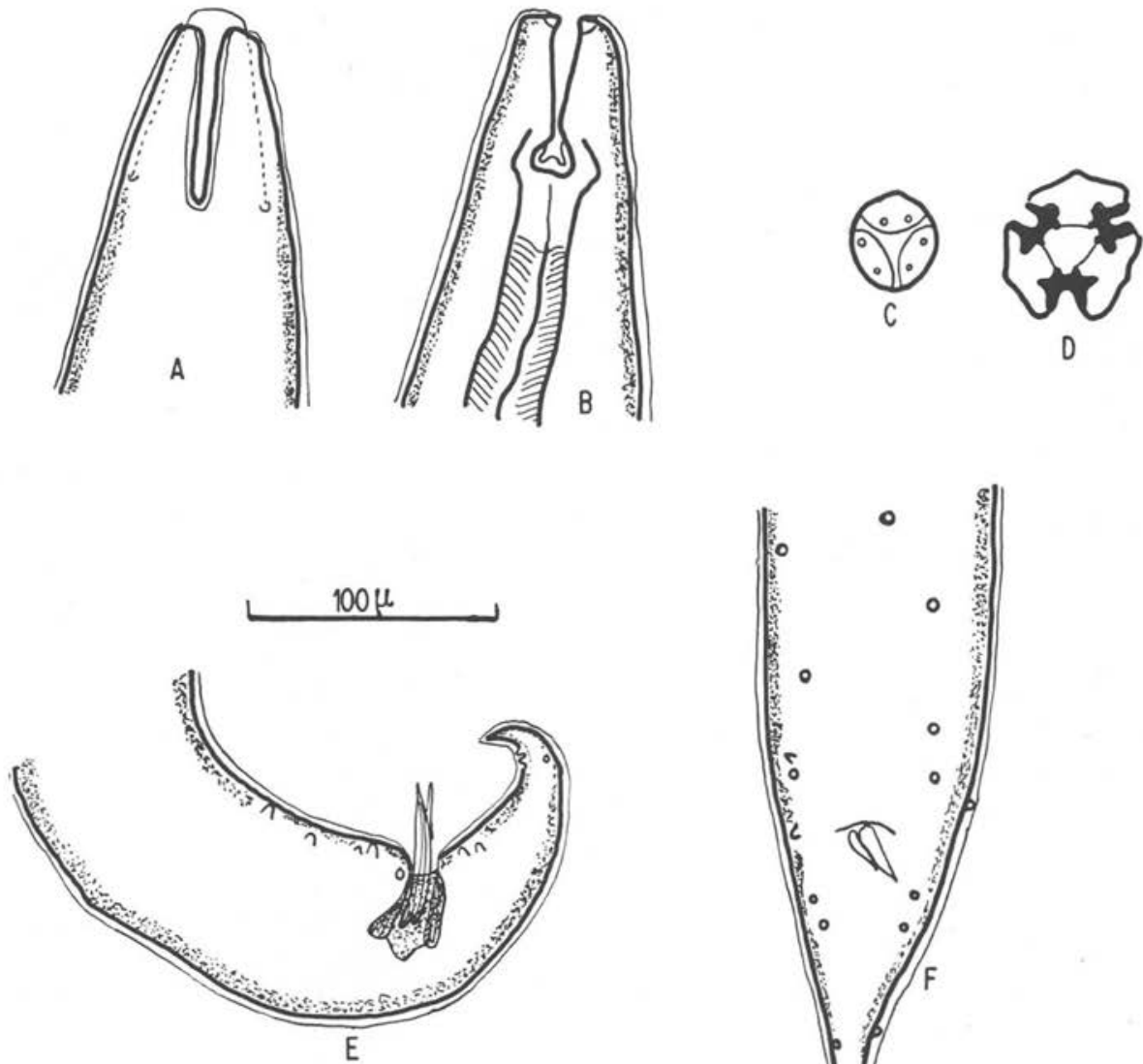

Maracaya graciai :

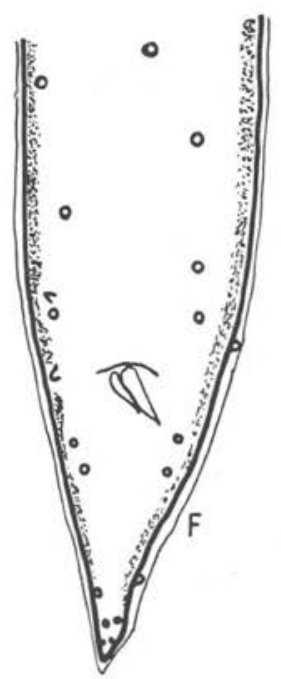

FIg. 4. - A : extrémité antérieure, vue subventrale superficielle. - B : extrémité antérieure, vue subventrale profonde. - C : extrémité antérieure, vue apicale superficielle. — D : extrémité antérieure, vue apicale au niveau du renforcement chitinoïde de la capsule buccale. E : extrémité postérieure du mâle, vue latérale. — F : extrémité postérieure du mâle, vue ventrale

de soutien chitinoïde, réunissant les bords des lèvres adjacentes, et ayant à peu près la forme d'un $\mathrm{H}$. Cette pièce forme par sa face externe la base du renforcement chitinoïde des lèvres et par sa face interne une ébauche de capsule buccale et le début du pharynx. 
La distance de ce support chitinoïde à l'apex est de $60 \mu$; il est donc situé en avant de la base des lèvres. Pharynx long de $40 \mu$. Esophage divisé en trois parties : antérieure musculaire, cylindrique, légèrement élargie à son tiers antérieur, moyenne en isthme rétréci, postérieure en bulbe valvulé. Pore excréteur au niveau du bulbe.

Mâle: Corps long de $1,46 \mathrm{~mm}$, large au maximum de $130 \mu$. Esophage musculaire de $450 \times 60 \mu$, isthme de $65 \mu$, bulbe de $105 \times 85 \mu$. Pore excréteur à $565 \mu$ de l'apex. Spicules égaux, longs de $60 \mu$. Gubernaculum long de $35 \mu$. Queue longue de $115 \mu$. Dix paires de papilles cloacales réparties comme l'indique la figure $4 \mathrm{~F}$.

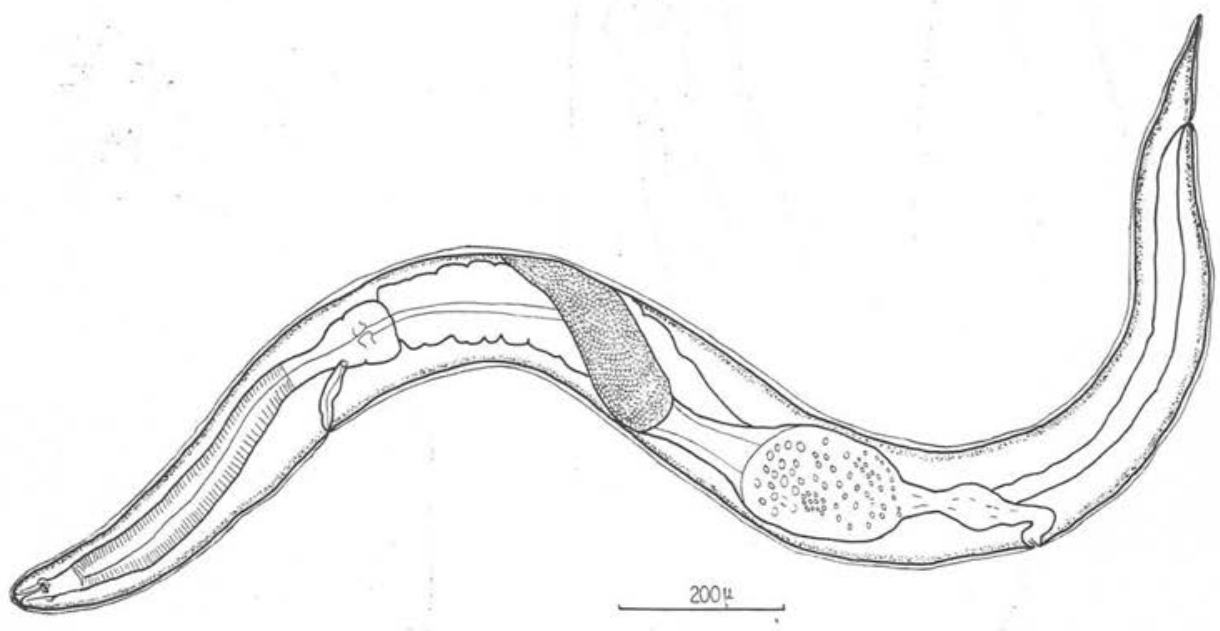

Maracaya graciai :

FIG. 5. - Femelle, organisation interne

Femelle : Corps long de $2 \mathrm{~mm}$, large au maximum de $160 \mu$. Esophage musculaire de $360 \mu \times 60 \mu$, isthme de $70 \mu$, bulbe de $75 \mu \times 80 \mu$. Pore excréteur à $460 \mu$ de l'apex. Vulve à $700 \mu$ de l'extrémité postérieure. Vagin long de $220 \mu$ se poursuivant vers l'avant par un utérus et un ovaire uniques. Queue longue de 125-160 $\mu$.

\section{Discussion :}

L'espèce se rattache à la famille des Atractidae par la monodelphie, la structure de l'œsophage et les caractéristiques de la queue du mâle. Selon la clef de Chabaud et Petter (1960), elle est assez proche du genre Ibrahimia Khalil 1932, mais s'en différencie par :

— la bouche : Ibrahimia a trois lèvres aiguës sans rebord chitineux et sans pièce de soutien postérieure,

- l'isthme œsophagien peu marqué chez Ibrahimia, 
- la vulve plus postérieure chez Ibrahimia (dernier sixième du corps et non un peu en arrière du second tiers).

Il est donc nécessaire de créer pour elle un nouveau genre et nous proposons la désignation de Maracaya graciai $\mathrm{n}$. gen., n. sp., le nom du genre rappelant celui de la localité où fut effectuée la récolte et le nom d'espèce étant dédié à notre collaborateur et ami le Docteur Angel Gracia Rodrigo, de Caracas.

La définition du genre est la suivante :

\section{Maracaya n. gen.}

Atractidae ayant une bouche à trois grandes lèvres qui présentent des renforcements chitinoïdes se réunissant en une pièce de soutien qui sert à la fois de capsule buccale et
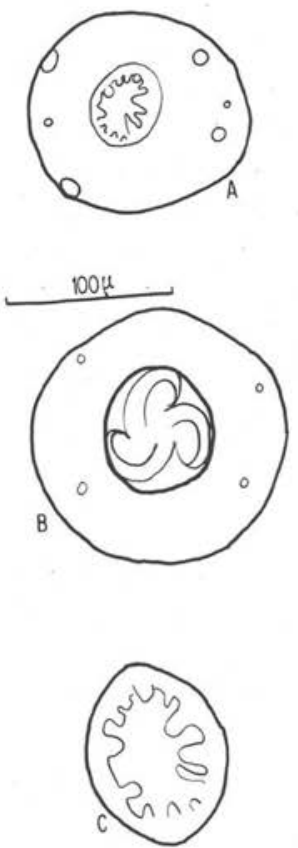

$100 \mu$
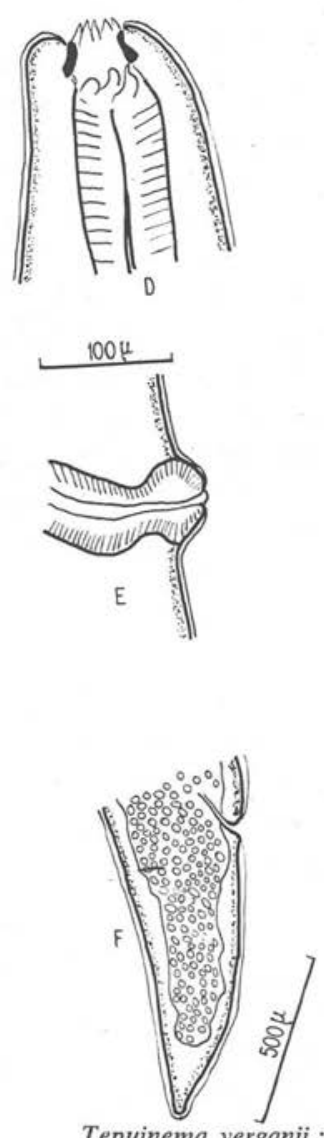

Tepuinema verganii :

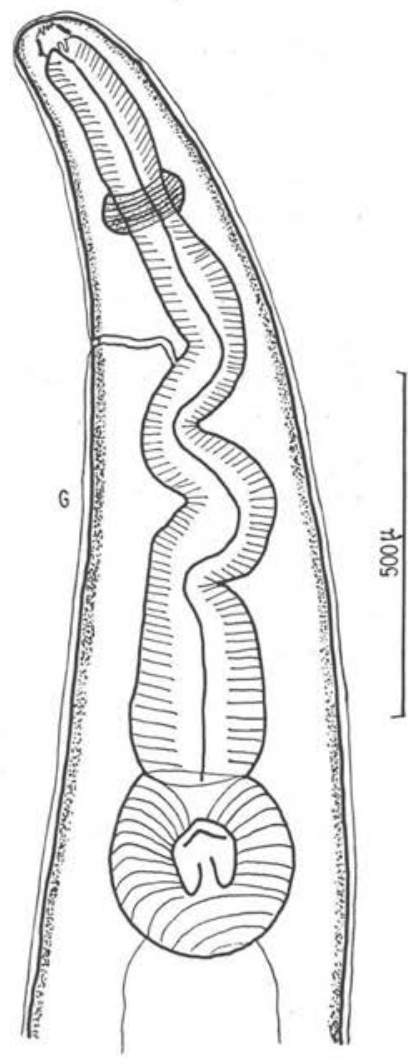

Fig. 6. - A: tête, vue frontale superficielle. - B: tête, vue frontale profonde, au niveau du pharynx. - C: détail des dents buccales, vue frontale. - D : tête, vue latérale. - E: détail de la vulve. - F : extrémité postérieure de la femelle. - G : extrémité antérieure de la femelle (A et B, même échelle; D et $\mathrm{E}$, même échelle). 
de pharynx antérieur. Esophage en trois parties : corpus, isthme bien différencié, et bulbe valvulé. Spicules égaux beaucoup plus long que le gubernaculum. Vulve postéquatoriale. Monodelphie. Parasites intestinaux de Reptiles. Espèce type unique : Maracaya graciai $\mathrm{n}$. $\mathrm{sp.}$

\section{4. - TEPUINEMA VERGANII n. gen., n. sp. *}

Nous n'avons que deux femelles provenant de l'intestin de Souris Mus musculus L., vivant en forêt à Uruyen (Auyentepui, Vénézuéla). Bien que le mâle ne soit pas connu il nous semble utile de décrire l'espèce, car la structure pharyngée indique qu'il s'agit d'un Subulurinae Travassos 1914, mais aucun des genres de la sous-famille n'a la même formation céphalique.

\section{Deseription :}

Corps robuste, légèrement rétréci aux extrémités, long de $18 \mathrm{~mm}$ et large au maximum de $670 \mu$. Capsule buccale à parois épaisses, surtout en arrière, haute de $28 \mu$ et ayant un diamètre de $38 \mu$ à sa partie la plus étroite, et de $53 \mu$ à sa partie la plus large. Il n'y a pas de lèvres, mais une bouche subcirculaire d'où émerge une sorte de coronule comparab'e à celle des Strongles. Celle-ci est formée de 12 éléments alternativement longs et courts. La tête porte quatre papilles submédianes et deux amphides latérales. Le fond de la cavité buccale est occupé par trois apophyses pharyngées disposées hélicoïdalement, avec les bords extérieurs chitinoïdes et le reste musculeux. Esophage long au total de $1,4 \mathrm{~mm}$, s'élargissant progressivement et se terminant par un bulbe valvulé de $270 \mu$. Anneau nerveux et pore excréteur respectivement à 320 et $460 \mu$ de l'apex. La vulve à $5,94 \mathrm{~mm}$ de l'extrémité antérieure présente deux petites lèvres légèrement saillantes et un ovéjecteur musculeux. Queue longue de $870 \mu$. Eufs in utero de $68 \times 38 \mu$.

\section{Discussion :}

La structure pharyngée et l'œsophage indiquent que l'espèce appartient aux Subulurinae Travassos 1914, mais la disparition des lèvres, remplacées par une coronule de 12 éléments n'est pas encore connue chez aucun Subulure et nécéssite la création d'un nouveau genre. Nous proposons de désigner notre matériel sous le nom de Tepuinema verganii $\mathrm{n}$. gen., n. sp., le nom de genre rappelant le site de la récolte, et le nom d'espèce étant dédié à notre collaborateur Franco Vergani.

Le genre est défini de la façon suivante: Tepuinema $\mathrm{n}$. gen.

Subulurinae avec capsule buccale à parois bien chitinoïdes, et bouche entourée d'une coronule de 12 éléments alternativement longs et courts. Parasites intestinaux de Rongeurs. Espèce type unique : Tepuinema verganii $\mathrm{n}$. sp., parasite de Mus musculus au Vénézuéla.

* Nous remercions vivement le $\mathrm{D}^{\mathrm{r}} \mathrm{W}$. Inglis d'avoir examiné ce matériel et d'avoir bien voulu nous donner son avis à ce sujet. 


\section{5. — TEJERAIA MEDIOSPIRALIS (Molin 1860) n. gen., n. comb. [ = Physocepha!us mediospiralis (Molin 1860) $]$ (fig. 7-8)}

Physocephalus mediospiralis, parasite de Dasyprocta agouti (L.), au Brésil a été décrit par Molin (1860), puis par Drasche (1883). Il n'y a aucune description moderne et le travail de Drasche comporte une erreur dans la numérotation des figures; il en résulte que dans les monographies classiques (Hall 1916) l'espèce est décrite avec une vue apicale correspondant à la tête de Serticeps vulvoinflata (Molin 1860).

Le matériel abondant que nous avons récolté dans l'estomac de Cuniculus paca (L.), du Territoire fédéral de l'Amazonie (Vénézuéla) nous permet de donner la redescription nécessaire et de préciser que l'ouvrage de Drasche comporte une bonne figure de l'extrémité céphalique de l'espèce (indiquant tout le système sensoriel céphalique et les dents internes) ; cette figure correspond au $\mathrm{n}^{\circ} 14$, pl. XII et non au $\mathrm{n}^{\circ} 16$ comme il est indiqué par erreur.

\section{Description :}

Corps allongé avec extrémité antérieure fine et extrémité postérieure obtuse. L'extrémité antérieure est couverte par une vésicule céphalique s'élargissant postérieurement pour se terminer un peu avant l'extrémité postérieure du pharynx, et un peu après le niveau des diérides ; celles-ci sont insérées sur deux manchons et leur pointe fait saillie à travers la vésicule. Les ailes cervicales longues de $2,54 \mathrm{~mm}$, naissent juste en arrière de la vésicule, s'élargissent rapidement, puis s'amoindrissent progressivement. Chaque aile est triple avec un élément central plus développé que les deux autres (fig. $8 \mathrm{C}$ ).

Tête avec deux lèvres latérales trilobées, séparées par deux larges échancrures (ventrale et dorsale). Quatre papilles latéro-médianes postérieures, deux amphides latérales et six papilles sur le cycle interne. En arrière de la bouche, deux fortes dents internes (dorsale et ventrale) correspondent aux échancrures des lèvres. La base des dents s'insère sur le pharynx en formant une courte capsule buccale dont les bords internes latéraux sont plissés longitudinalement, ce qui donne à la paroi une apparence rugueuse. Pharynx long, formé de 25 anneaux chitinoïdes disposés en spirale. Esophage divisé en une portion musculaire courte et une portion glandulaire longue. Pore excréteur et anneau nerveux à la partie moyenne de l'œsophage musculaire.

Mâle: Long de $30 \mathrm{~mm}$, large au maximum de $500 \mu$. Cavité buccale longue de $36 \mu$, pharynx de 270-390 $\mu$, œsophage musculaire de 370-495 $\mu$, œsophage glandulaire de 3,00 à $3,30 \mathrm{~mm}$. Diérides, anneau nerveux et pore excréteur respectivement à 245$260 \mu, 440-535 \mu$ et 496-516 $\mu$ de l'apex. Ailes cervicales longues de 2,54 mm. Extrémité postérieure fortement courbée, avec des ailes caudales à peu près symétriques, à surface ventrale ornée d'une cuticule finement pavimenteuse. 21 papilles et 2 phasmides subterminales dont la répartition est indiquée sur les figures $\mathrm{B}$ et $\mathrm{D}$. Spicules très inégaux : droit long de 2,51-2,65 mm, gauche peu chitinoïde long de 68-80 $\mu$. Gubernaculum granuleux de petite taille. 
Femelle : Longueur $40 \mathrm{~mm}$, largeur maxima $750 \mu$. Cavité buccale, pharynx, œsophage musculaire et œsophage glandulaire longs respectivement de $40 \mu, 290-400 \mu$, 450-507 $\mu$ et 3,1-3,5 mm. Diérides à 260-300 $\mu$ de l'apex. Anneau nerveux et pore excréteur au même niveau à 500-550 $\mu$. Ailes cervicales longues de 2,7 mm. Queue de $160-190 \mu$. Vulve à $1,50-1,55 \mathrm{~mm}$ de l'extrémité postérieure, avec un long ovéjecteur $(7,62 \mathrm{~mm})$ dirigé vers l'extrémité antérieure.
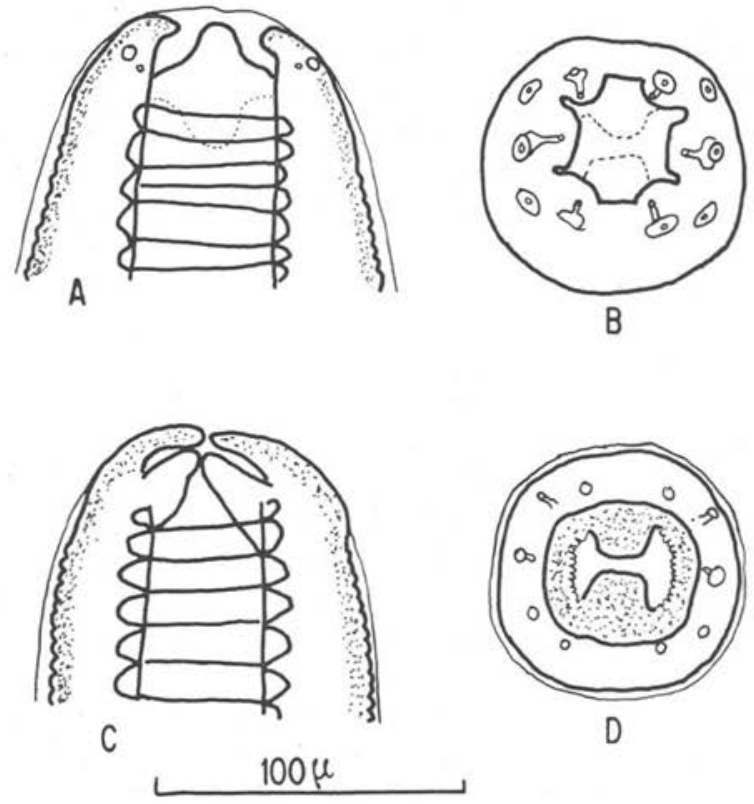

Tejeraia mediospiralis :

Fig. 7. - A : extrémité antérieure, vue dorsoventrale. B: extrémité antérieure, vue frontale au niveau du cycle interne. - C: extrémité antérieure, vue latérale. - D: extrémité antérieure, vue frontale au niveau de la capsule buccale

\section{Discussion :}

Bien qu'ayant beaucoup de caractères communs avec les espèces du genre Physocephalus, la forme décrite plus haut s'éloigne profondément de ce genre par les lèvres beaucoup plus faibles, la présence de dents médianes dans la capsule buccale, et, accessoirement par la position plus postérieure de la vulve. Il nous semble donc nécessaire de la placer dans un genre nouveau pour lequel nous proposons le nom de Tejeraia, en hommage au Docteur E. Tejera, pionnier de la parasitologie vénézuélienne. L'intérêt de cette forme vient de ce qu'elle est proche du genre Physocephalus, mais plus primitive en raison des lèvres latérales plus faibles et de la présence de dents médianes inter- 


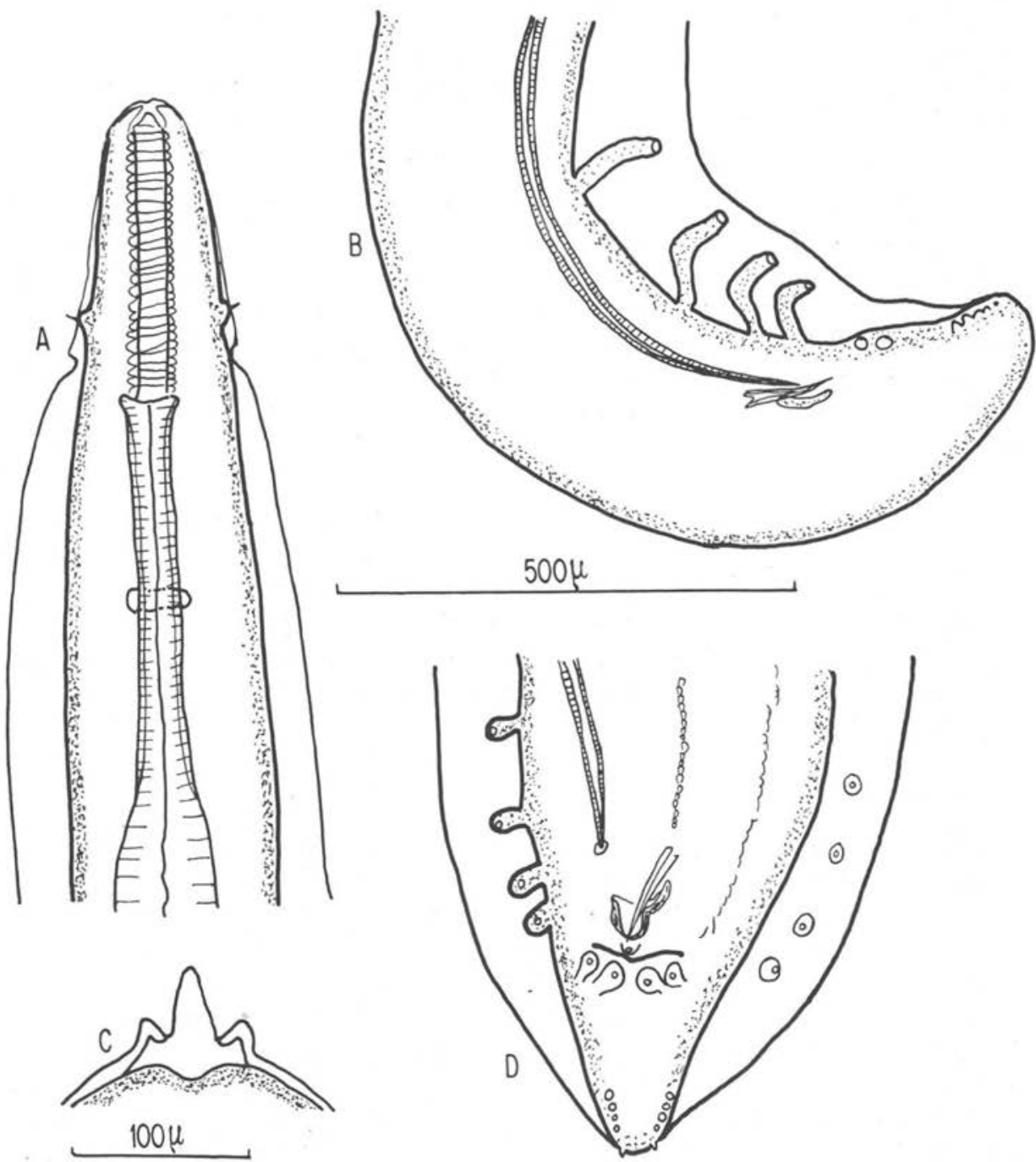

Tejeraia mediospiralis:

Fig. 8. - A : extrémité antérieure, vue médiane. - B : extrémité caudale du mâle, vue latérale. C: Coupe transversale au niveau des ailes cervicales. - D : extrémité caudale du mâle, vue ventrale.

nes correspondant vraisemblablement à l'invagination des reliefs médians très fréquents chez les larves de Spirurides.

Chabaud $(1959 a)$ a signalé le parallélisme entre Ascaropsinae et Spirurinae, remarquant que plusieurs genres d'Ascaropsinae correspondent point pour point à des Spirurinae, dont ils dérivent très probablement. C'est ainsi que le genre Protospirura 
(Spirurinae) correspond à Pygarginema (Ascaropsinae), le genre Hartertia (Spirurinae) correspond à Physocepha!us (Ascaropsinae), le genre Cylicospirura (Spirocercinae) correspond à Streptopharagus (Ascaropsinae). A cette liste nous pouvons ajouter que le genre Tejeraia (Ascaropsinae) correspond à Cyathospirura (Spirurinae).

Nous proposons la définition suivante: Tejeraia n. g.

Ascaropsinae: Cuticule cervicale dilatée. Diérides symétriques au niveau de la partie postérieure du pharynx. Ailes cervicales latérales triples, la lame centrale plus saillante. Bouche avec deux lèvres latérales trilobées et deux dents internes dorso-ventrales. Pharynx à peu près cylindrique, avec parois annelées en spirale. Mâle avec spicules très inégaux, gubernaculum petit et granuleux. Femelle avec vulve postérieure.

Parasites de l'estomac de Mammifères. Espèce type unique : Tejeraia mediospiralis (Molin, 1860), n. comb., parasite de Dasyprocta agouti (L.), Cuniculus paca (L.), et Tapirus americanus, Brisson.

\section{6. - PARABRONEMA BONNEI (Van Thiel 1925) Baylis et Daubney 1926 (= Squamanema bonnei Van Thiel 1925) (fig. 9)}

L'espèce fut décrite chez un Singe hurleur Alouatta seniculus L. (= Mycetes seniculus), de Surinam, par Van Thiel en 1925. Nous l'avons retrouvée abondamment dans l'intestin d'Alouatta ursina Humboldt, de Atures (Territoire fédéral de l'Amazonie, Vénézuéla).

\section{Description :}

Corps assez long, avec extrémité postérieure arrondie chez la femelle, incurvée et munie d'ailes caudales chez le mâle. Bouche entourée par deux pseudo-lèvres latérales saillantes en avant, à bord antérieur concave et par deux lèvres, ventrale et dorsale, entières plus basses que les pseudo-lèvres, et émettant chacune en profondeur une forte dent interne limitant l'ouverture du pharynx. Deux papilles submédianes sur chaque lèvre et une amphide sur chaque pseudo-lèvre. Les deux lèvres et les deux pseudo-lèvres font saillie postérieurement et constituent ainsi quatre écussons. Ceux-ci portent de profondes incisions transversales à leur deuxième tiers postérieur ; au-delà de l'incisure les écussons dorsaux et ventraux se divisent par une fente longitudinale en deux lames, alors que les écussons latéraux restent simples. Donc, la partie postérieure de l'armature céphalique porte six pièces chitinoïdes. Pharynx très long, diérides symétriques, un peu en arrière de l'anneau nerveux. Cuticule dilatée asymétriquement ainsi que l'a déjà noté Van Thiel.

Femelle: Corps long de 19-25 mm, large au maximum de 250-280 $\mu$. Ensemble des écussons céphaliques formant un trapèze haut de $52 \mu$ et large de $70 \mu$ à la base. Pharynx long de $230 \mu$, œsophage de 1,02 mm. Pore excréteur, anneau nerveux et diérides respectivement à $260 \mu, 290 \mu$ et $300-320 \mu$. Vulve un peu saillante à $3,47 \mathrm{~mm}$ de l'extrémité postérieure. Queue longue de 220-280 $\mu$. 
Mâle: Corps long de 12,5-15 mm, large au maximum de 175-190 $\mu$. Pharynx long de 165-205 $\mu$. Esophage long de 1,12-1,24 mm et large au maximum de $65 \mu$. Diérides, pore excréteur et anneau nerveux aux mêmes niveaux que chez la femelle.
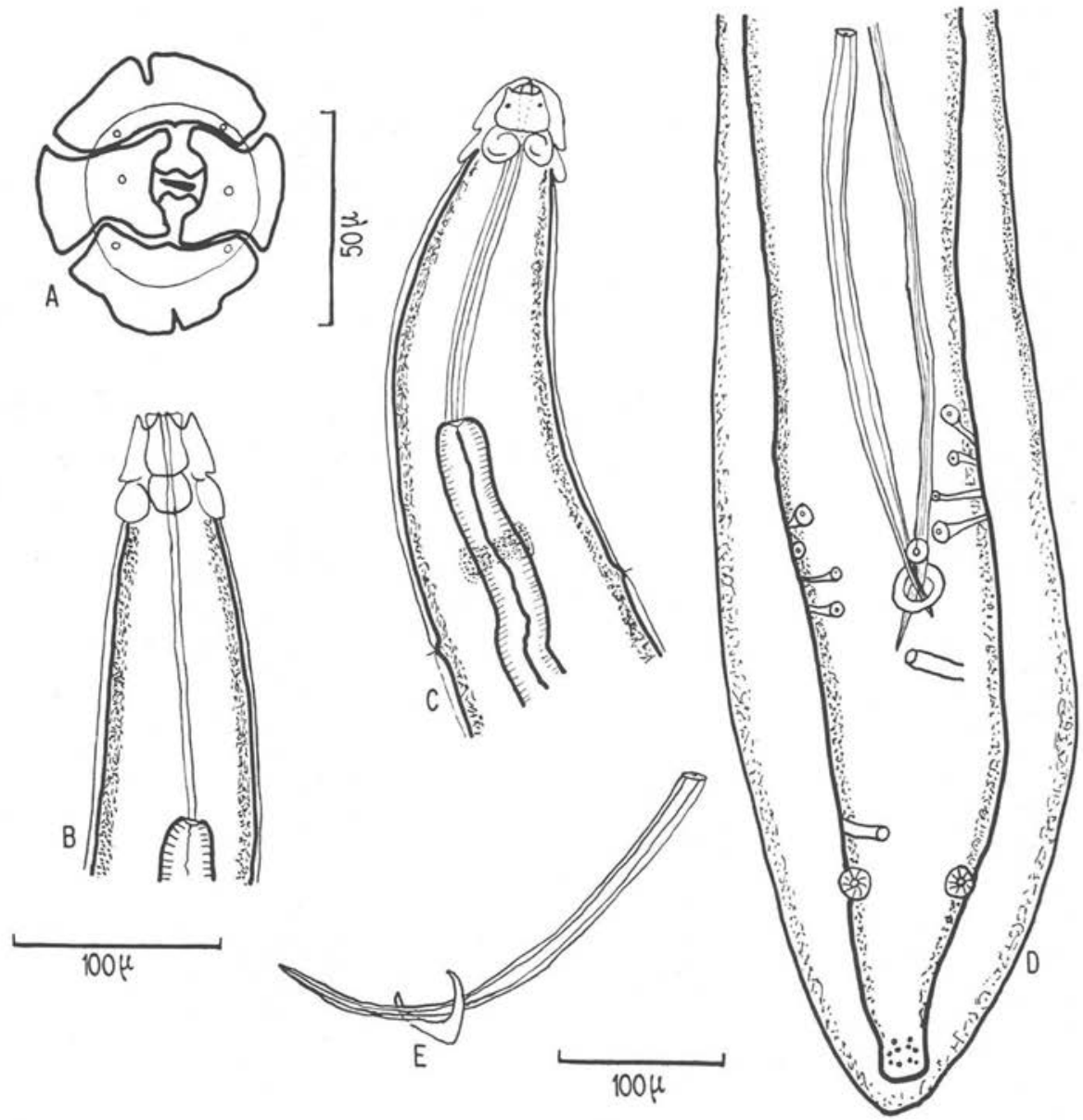

Parabronema bonnei :

Fig. 9. - A : extrémité céphalique, vue frontale. -- B : extrémité antérieure, vue latérale. C: extrémité antérieure, vue ventrale. - D : extrémité postérieure du mâle, vue ventrale. $\mathrm{E}$ : Gubernaculum et petit spitule (C, D et $\mathrm{E}$ même échelle)

Ailes caudales inégales, la plus longue atteignant presque $1 \mathrm{~mm}$. Spicules inégaux, le droit long de $310-385 \mu$, le gauche $1,97-2,25 \mathrm{~mm}$. Gubernaculum petit. 21 papilles et deux phasmides réparties comme l'indique la figure $9 \mathrm{D}$. Queue longue de $310 \mu$. 


\section{Discussion :}

La description originale date de 1925 et ne comporte pas l'étude de la tête en vue apicale. La place générique de l'espèce a été fréquemment discutée. Nous trouvons une structure buccale parfaitement conforme à celle du genre Parabronema (cf. Chabaud, 1958), et n'hésitons donc pas à confirmer la synonymie du genre Squamanema.

\section{7. - APROCTA GOLVANI n. sp. (fig. 10)}

Le matériel a été récolté dans les séreuses de Monasa atra (Boddaert), du Territoire fédéral de l'Amazonie (Vénézuéla).

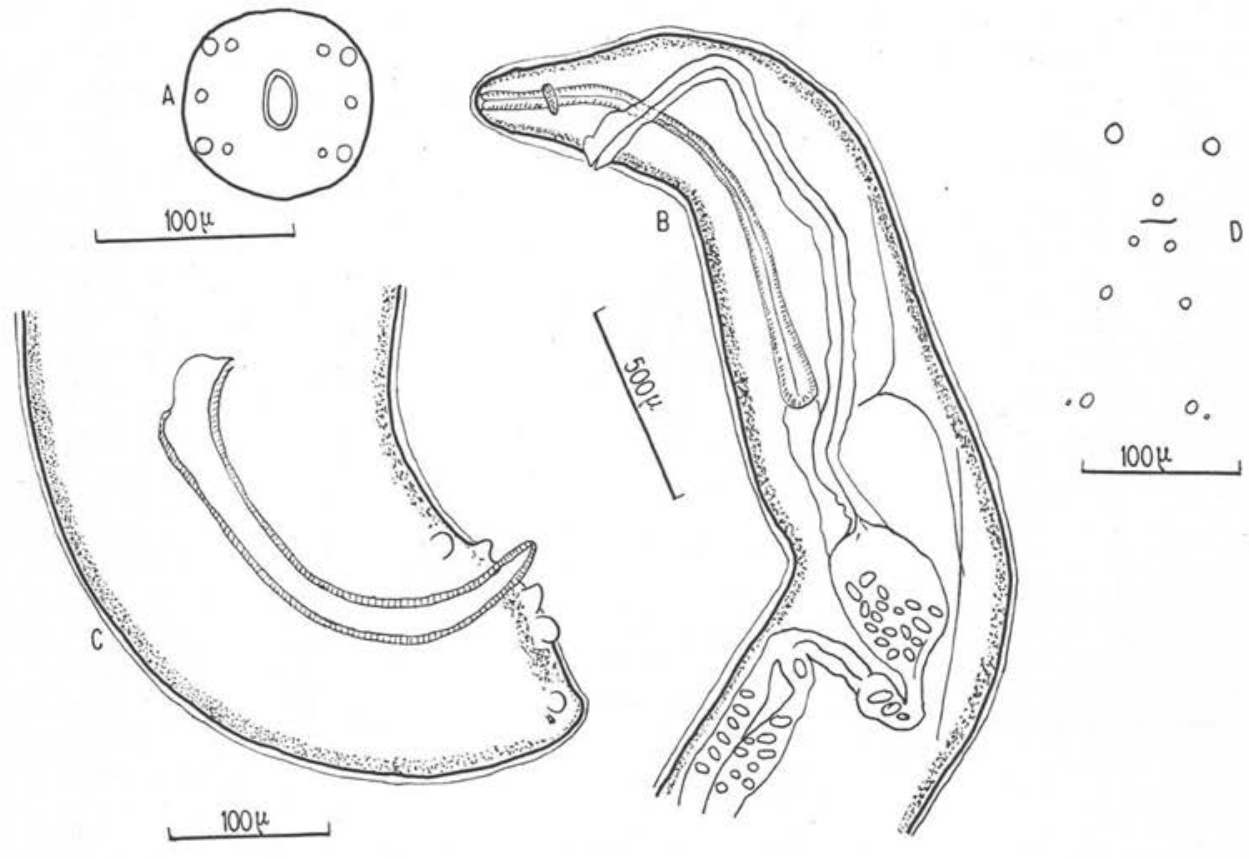

Aprocta golvani :

FIG. 10. - A : tête, vue apicale. - B : extrémité antérieure de la femelle. — C : extrémité postérieure du mâle, vue latérale. - D : Disposition des papilles génitales du mâle, vue ventrale.

\section{Description :}

Tête arrondie avec bouche ovalaire bordée par un renforcement chitinoïde simulant une capsule buccale. Huit papilles sur le cycle externe, les quatre latéromédianes plus grandes et plus postérieures que les médiomédianes et deux amphides latérales. Esophage non divisé, cylindrique et légèrement enflé postérieurement. 
Mâle: Corps long de 10,4 mm, large au maximum de $490 \mu$. Anneau nerveux à $150 \mu$ de l'apex. Queue obtuse, longue de $100 \mu$. Spicules égaux, courts et robustes, longs de $300 \mu$. 9 papilles (1 paire et 1 impaire précloaca'es, 3 paires postcloacales s'écartant progressivement) et une paire de phasmides subterminales.

Femelle: Corps long de $17 \mathrm{~mm}$, large au maximum de $650 \mu$. Esophage long de $1,26 \mathrm{~mm}$, large de $100 \mu$. Anneau nerveux à $185 \mu$ de l'apex. Vulve à $325 \mu$ de l'extrémité antérieure, munie d'un long ovéjecteur dont la partie terminale se dilate en un réceptacle impair précédant la division en deux utérus. La longueur totale de l'ovéjecteur est de $2,5 \mathrm{~mm}$. Eufs in utero longs de $70 \mu$ et larges de $35 \mu$.

\section{Discussion :}

Les caractères céphaliques sont souvent trop mal connus pour permettre une différenciation des nombreuses espèces décrites dans le genre Aprocta, et nous devons nous baser essentiellement sur les dimensions des spicu'es et le nombre des papilles cloacales. Trois espèces paraissent particulièrement proches de la nôtre.

A. caprimulgi (Kazulski, 1958) se distingue facilement par son œsophage divisé en deux parties et surtout par sa grande cavité buccale.

A. semenovi Skrjabin 1934 se différencie de la nôtre par le nombre de papilles cloacales et les caractères céphaliques.

Enfin A. nyctidromi Caballero et Peregrina 1938 a un œsophage divisé en deux parties, et les spicules, d'après la figure des auteurs, semblent longs de $940 \mu$ et non de $300 \mu$. Nous pensons donc que l'espèce décrite plus haut est nouvelle et nous proposons le nom de Aprocta golvani n. sp. en hommage à notre Professeur et ami Y.-J. Golvan, Professeur agrégé à la Faculté de Médecine de Paris.

\section{8. - LITOMOSOIDES BRASILIENSIS Lins de Almeida 1936 (fig. 11)}

Chez différents Chiroptères du Vénézuéla : Myotis sp. de Caracas, Phyllostomus sp. de Cabure (Etat de Falcon), Carollia perspicillata L. de Tiara (Etat d'Aragua) et de Caura (Etat de Bolivar), nous avons trouvé une filaire parasite de la cavité générale, que nous identifions à Litomosoïdes brasiliensis Lins de Almeida 1936.

\section{Description :}

Pharynx chitinoïde long de $23 \mu\left(\delta^{\zeta}\right)$ ou $27 \mu(\$)$, présentant deux épaissisements circulaires, le postérieur étant le plus développé. CEsophage un peu dilaté en avant pour envelopper la base du pharynx, et non divisé en deux parties.

Mâle: Corps long de $50 \mathrm{~mm}$, large au maximum de $220 \mu$. Esophage de $780 \times 50 \mu$. Spicule gauche long de 480-490 $\mu$ divisé en un manche et une lamina filiforme. Spicule droit long de 142-168 $\mu$, robuste avec extrémité clistale chitinoïde en forme de pince. Queue longue de 270-280 $\mu$. L'extrémité postérieure, en vue latérale, montre 8 papilles, et nous pensions au premier examen qu'il y avait 8 paires de papilles ; mais, le même exemplaire examiné en vue ventrale a montré que les papilles sont ali- 
gnées sur une file longitudinale médio-ventrale et qu'il n'y a pas 8 paires de papilles, mais seulement 4 paires et deux phasmides. Cela coïncide avec la figure de Lins de Almeida qui montre clairement 2 séries de quatre papilles. Un autre exemplaire examiné montre

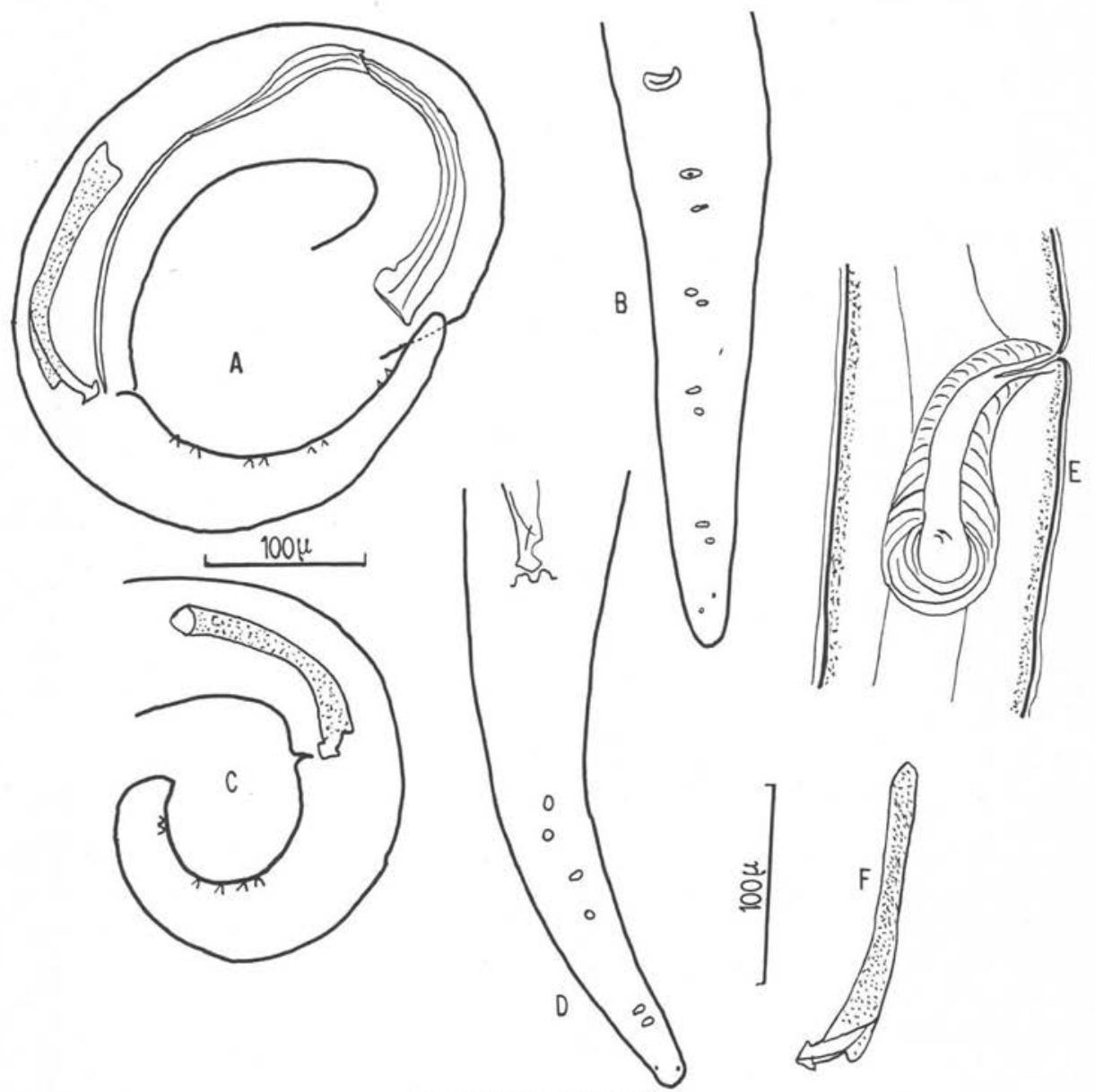

Litomosoides brasiliensis:

Fig. 11. - A : mâle, extrémité postérieure, vue latérale. - B : mâle, vue ventrale. - C : vue latérale d'un autre mâle, montrant huit papilles. - D : vue ventrale du même. - E : vulve et ovéjecteur. $\mathrm{F}$ : petit spicule isolé (A, C et E, même échelle; B, D et F, même échelle).

également 4 paires de papilles, mais la paire antérieure est juste en arrière du cloaque et n'est pas visible en vue latérale.

Femelle : Corps long de $110 \mathrm{~mm}$, large au maximum de $200 \mu$. Esophage long de $1,25 \mathrm{~mm}$, large à la base de $60 \mu$. Anneau nerveux à $85 \mu$ de l'apex. Vulve à 2,2-3,0 mm 
de l'extrémité antérieure. Ovéjecteur très musculeux, long de $180 \mu$, large de $75 \mu$, suivi de deux utérus. Queue longue de $370 \mu$.

\section{Discussion :}

Huit espèces sont décrites dans le genre Litomosoïdes, et sont différenciées par les caractères des spicules et le nombre de papilles cloacales. Celles-ci, malheureusement, ont toujours été étudiées uniquement sur des vues latérales, qui, ainsi que nous l'avons vu plus haut, prêtent facilement à erreur. Nous ne voyons aucune différence valable entre notre matériel, la description de L. brasiliensis Lins de Almeida 1936 et la description de L. caballeroi Gracia Rodrigo 1960. Nous pensons donc que cette dernière est synonyme de $L$. brasiliensis. En 1961 Rego a réétudié $L$. brasiliensis et a proposé son identité avec $L$. carolliae Caballero 1944. Nous ne pensons pas que cette seconde synonymie puisse être admise, car Rego a observé une variation du nombre de papilles allant de 5 à 10 paires et a pensé que Lins de Almeida n'avait pas observé la $5^{\circ}$ paire. Nous confirmons ici l'observation de ce dernier auteur, et nous croyons qu'il serait nécessaire d'étudier les queues du mâle des différentes espèces en vue ventrale, avant d'admettre cette synonymie.

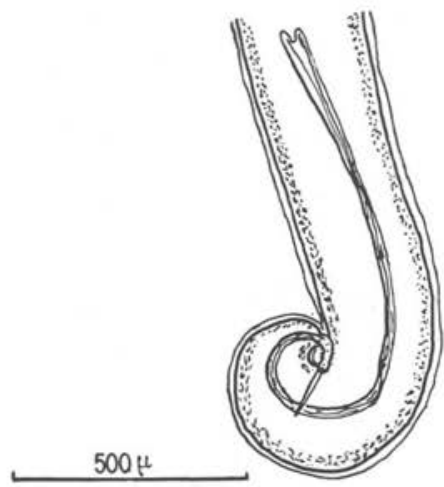

Trichuris felis :
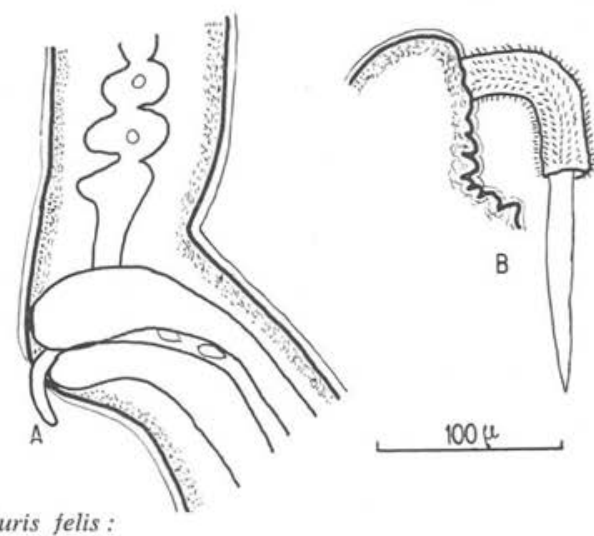

Fig. 13. - A : région de la vulve. - B :
détail de la gaine du spicule
Fig. 12. - A: vue générale de l'extrémité postérieure du mâle
Fig. 13. - A: région de la vulve. - B :
détail de la gaine du spicule

\section{9. - TRICHURIS FELIS (Diesing 1851)}

$$
\text { (=T. serratus Linstow } 1879 \text { sensu Cameron 1937) (fig. 12-13) }
$$

Les exemplaires que nous avons récoités chez Felis tigrina Scheber, au Vénézuela, ont l'intérêt de provenir du même hôte que les exemplaires décrits par Diesing (1851) sous le nom de Trichuris felis. Le nom donné par Diesing a été rejeté par certains auteurs qui estiment que la description de Diesing est insuffisante, mais nous ne pensons pas que Trichocephalus felis puisse être considéré comme nomen nudum, puisque l'auteur a précisé l'hôte et a donné quelques lignes de description. Nous croyons donc 


\begin{tabular}{|c|c|c|c|c|c|c|c|c|c|c|}
\hline 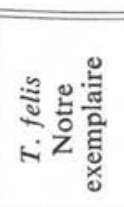 & 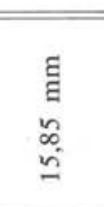 & 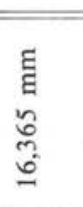 & 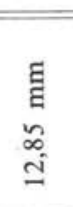 & 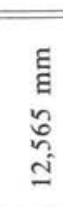 & 鼠 & 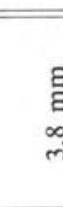 & & $f$ & $\vec{m}$ & 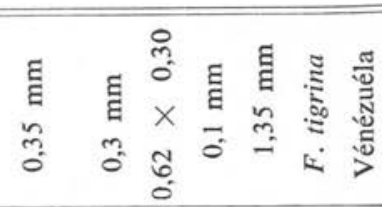 \\
\hline 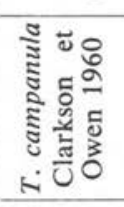 & 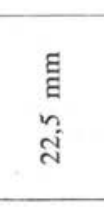 & 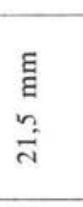 & 1 & I & I & I & & $\frac{\sigma}{f}$ & $\bar{m}$ & 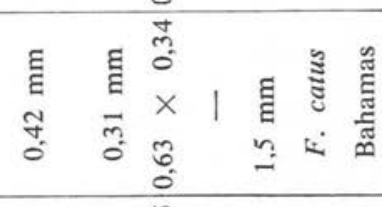 \\
\hline 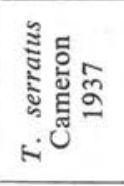 & 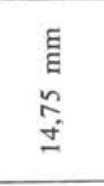 & 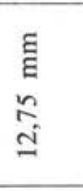 & $\begin{array}{l}\text { 压 } \\
\text { = }\end{array}$ & 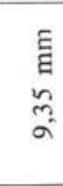 & 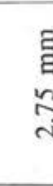 & J & & r & $\bar{m}$ & 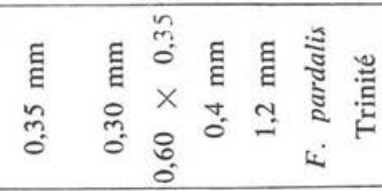 \\
\hline 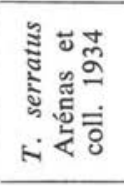 & 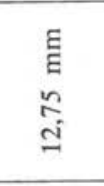 & 1 & 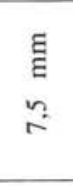 & 1 & . & ह & & $n$ & 1 & 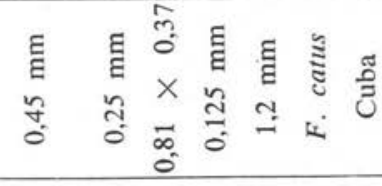 \\
\hline 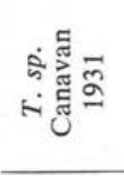 & $\begin{array}{l}\text { 晨 } \\
q\end{array}$ & 1 & 1 & 1 & I & 1 & & & 1 & 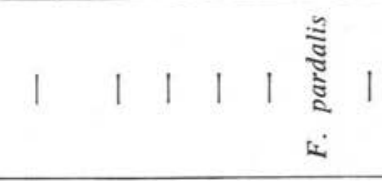 \\
\hline 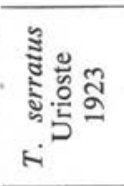 & $\begin{array}{l}\text { ह } \\
\text { ॠ }\end{array}$ & 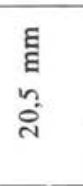 & $\stackrel{E}{\underline{E}}$ & 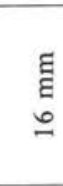 & 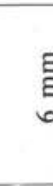 & 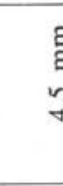 & & $\infty$ & $\sqrt{5}$ & 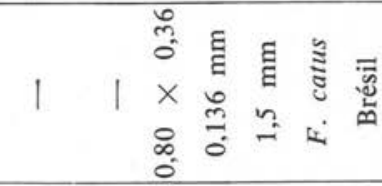 \\
\hline 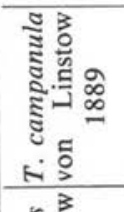 & $\begin{array}{l}\frac{E}{E} \\
\frac{n}{m}\end{array}$ & 1 & 1 & 1 & I & 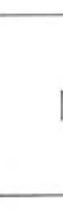 & & f & $\bar{\xi}$ & 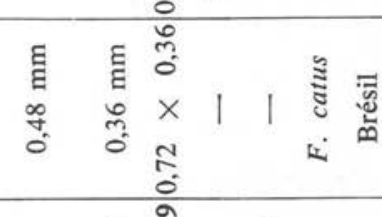 \\
\hline 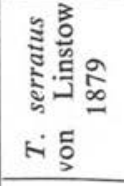 & $\begin{array}{l}\text { 茞 } \\
\text { 号 }\end{array}$ & $\begin{array}{l}\text { 音 } \\
\text { 吕 }\end{array}$ & 1 & 1 & 1 & 1 & & ה & สิ & 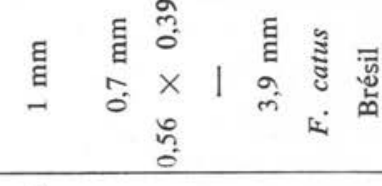 \\
\hline 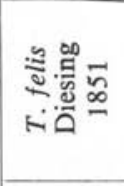 & 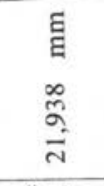 & I & 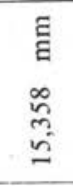 & 1 & & & & & 1 & 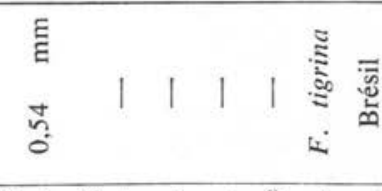 \\
\hline & 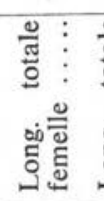 & 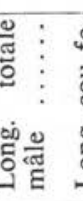 & & & & & & & & 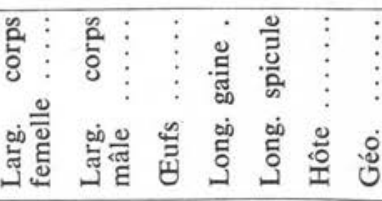 \\
\hline
\end{tabular}


qu'il est utile de donner les dimensions de nos spécimens (tableau) et les figures concernant la région vulvaire de la femelle et l'extrémité postérieure du mâle (fig. 12 et 13).

La systématique des Trichuris de Félidés américains est complexe. En 1879, von Linstow décrit Trichocephalus serratus chez le chat domestique du Brésil, puis en $1889 T$. campanula, chez le même hôte de la même région. En 1923, Urioste examine des exemplaires de même provenance et estime que les deux espèces de Linstow sont identiques. En 1931, Canavan rencontre des Trichuris chez Felis pardalis (L.), mort en captivité aux Etats-Unis. Il ne donne pas la longueur des femelles et ne propose pas de détermination spécifique. En 1937, Cameron étudie des spécimens trouvés chez Felis pardalis de la Trinité et les nomme $T$. serratus. En 1960, Clarkson et Owen décrivent comme Trichuris sp., les exemplaires provenant de Chats des Iles Bahamas. Au Vénézuela, Vogelsang (1940) a signalé a deux reprises $T$. campanula dans le cæcum du chat domestique ; Vogelsang et Mayaudon ont identifié à la même espèce des Trichocéphales provenant de l'csophage et de l'aorte du Canidé Cerdocyon thous thous (L.), de Zaraza.

Nous donnons un tableau des mensurations publiées par les différents auteurs (la longueur de la gaine donnée par Cameron diffère des trois autres, mais l'auteur s'est basé sur une gaine invaginée).

Nos spécimens provenant du même hôte Felis tigrina que ceux de Diesing doivent être nommés Trichuris felis (Diesing) ; ils correspondent bien à la description de Cameron basés sur le parasite de Felis pardalis. Il reste à savoir si les formes trouvées chez le Chat domestique sont différentes et peuvent être nommées $T$. serratus, ou si elles sont identiques et doivent être nommées $T$. felis. L'examen du tableau ne permet pas de répondre nettement à cette question ; un matériel abondant et une comparaison avec des spécimens provenant de Chats domestiques semblent nécessaires pour résoudre le problème.

\section{Résumé}

Etude de neuf Nématodes parasites de Vertébrés vénézuéliens.

I. Triodontophorus nipponicus Yamaguti 1943, connu seulement d'ExtrêmeOrient, a été récolté chez le Cheval domestique et l'Ane au Venezuela. T. hsiungi K'ung, 1958 est mis en synonymie.

II. Longistriata dollfusi n. sp., parasite de Mus musculus, est remarquable par la dilatation cuticulaire de l'extrémité postérieure de la femelle et par l'hypertrophie de la côte antéro-latérale de la bourse caudale du mâle.

III. Maracaya graciai n. gen., n. sp., parasite d'Amphisbaena alba, est un Atractide présentant une curieuse pièce de soutien chitinoïde réunissant les bords labiaux et le début du pharynx.

IV. Tepuinema verganii n. gen., n. sp., parasite de Mus musculus, est un Subulurinae dont les lèvres sont remplacées par une coronule de 12 éléments alternativement longs et courts. Le mâle n'est pas connu. 
V. Spiroptera mediospiralis Molin 1860, a été retrouvé chez Cuniculus paca. L'espèce a de nombreuses analogies avec le genre Physocephalus, dans lequel elle avait été classée, mais la structure buccale est proche de celle du genre Cyathospirura. Nous proposons donc pour elle le nouveau genre Tejeraia (Ascaropsinae).

VI. Parabronema bonnei (Van Thiel 1925) est redécrit à partir de spécimens récoltés chez Alouatta ursina. La structure céphalique est conforme à celle du genre Parabronema et la mise en synonymie du genre Squamanema est confirmée.

VII. Aprocta golvani n. sp., parasite de Monasa atra, est séparé des espèces les plus proches par les caractères de l'extrémité postérieure du mâle.

VIII. Litomosoides brasiliensis Lins de Almeida 1936, récolté chez plusieurs Chiroptères est redécrit en insistant sur une disposition médio-ventrale des papilles cloacales, qui semble à l'origine de certaines erreurs. L. caballeroi Gracia Rodrigo 1960 est considéré comme synonyme de brasiliensis, alors que L carolliae Caballero 1944 semble valide.

IX. Trichuris felis (Diesing 1851) ne peut pas être considéré comme nomen nudem. Le matériel que nous avons étudié provient de l'hôte type et correspond au T. serratus Linstow sensu Cameron 1937, provenant de Felis pardalis. La revue des documents trouvés dans la littérature ne nous permet pas de savoir si le $T$. serratus Linstow 1879, parasite du Chat domestique, est identique ou différent.

\section{Bibliographie}

Arenas (R.), Kouri (P.) et Basnuevo (J. G.), 1934. - Sobre parasitisme en los animales domesticos. Bol. Col. Vet. Nal., Habana, III, 30, 764-768.

Artigas (P.) et Pacheco (G.), 1933. - Longistriata maldonadoi n. sp. (Nematoda), Trichostrongylide parasite du Myocastor coipus C.R. Soc. Biol., CXII, 1 004-1 006.

Caballero (E.) et Cerecero (M. C.), 1942. - Longistriata convoluta n. sp. (Nematoda, Trichostrongylidae) parasito del intestino de una «tuza $\$$ Cratogeomys merriami (Tho. mas). Anales del Inst. de Biol., XIV, 201-205.

— et Peregrina (D. I.), 1938. - Contribucion al conocimiento de los Nematodes de las Aves de Mexico, VII. Anal. Inst. Biol., IX, 151-163.

Cameron (T. W. M.), 1937. - Studies on the endoparasitic fauna of Trinitad Mammals, V. Further parasites from the ocelot. Canad. Jl. of Research, D, XV, 24-27.

Canavan (W. P. N.), 1931. - Nematode parasites of Vertebrates in the Philadelphia Zoological Garden and vicinity, II. Parasitol., XXIII, 196-229.

Chabaud (A. G.), 1958. - Essai de classification des Nématodes Habronematinae. Ann. Parasit., XXXIII, 445-508.

-, 1959. - - a) Phénomène d'évolution régressive des structures céphaliques et classification des Nématodes Spiruroidea. Parassit., I, 11-20.

—, 1959. - b) Sur la systématique des Nématodes proches de Spirocerca lupi (Rud. 1809). Parassit., I, 129-135.

—, 1959. - c) Remarques sur la systématique des Nématodes Trichostrongyloidea. Bull. Soc. Zool. France, LXXXIV, 473-483. 
-, Anderson (R.) et Brygoo (E. R.), 1959. - Sept Filaires d'Oiseaux malgaches. Ann. Parasit., XXXIV, 88-109.

- et Petter (A. J.), 1960. - Sur les Nématodes Atractides. Libro homenaje al $D^{r}$ E. Caballero, Mexico, 465-470.

ClaAkson (M. J.) et Owen (L. N.), 1960. - The species of Trichuris in the domestic cat. Jl. of Helminth., XXXIV, 319-322.

Diaz-Ungria (C.) et Vergani (F.), 1952. - Contribucion al conocimiento del genero Triodontophorus (Nematoda) en los equinos de Venezuela. Bol. Inst. Inv. Vet., IV, 20, 673710.

Diesing (K. M.), 1851. - Systema Helminthum. Berlin. 2 vol., 588 pp.

DRASCHE (R.), 1882-1883. - Revision der in der Nematoden-Sammlung des K.K. zoologischen Hofcabinetes befindlichen Original Exemplare Diesing's und Molin's. Verh. k.k. Zool. Bot. Ges. Wien., XXXII, 117-138; XXXIII, 107-118 et 193-218.

Gracia-Rodrigo (A.), 1960. - Contribucion al estudio de los Filarioidea de Quiroptera, con description de Litomosoides caballeroi $\mathrm{n}$. sp. Thèse. Caracas.

Hall (M. C.), 1916. - Nematode parasites of Mammals of the orders Rodentia, Lagomorpha and Hyracoidea. Proc. U.S. Nat. Mus. Washington, XX, 259 pp.

INGLIS (W. G.), 1958. - The comparative anatomy of the Subulurid head (Nematoda), with a consideration of its systematic importance. Proc. Zool. Soc. London, CXXX, $577-$ 604.

KhalIL (M. B.), 1932. - Parasites from Liberia and French Guinea. First Part, Nematoda. Zeit. Parasit., IV, 431-458.

K'UNG (F. Y.), 1958. - Triodontophorus hsungi n. sp. a new Nematode parasite of donkeys. Acta Vet. et Zool. Sinica, III, 14-18 (en chinois, résumé en anglais).

Lins DE AlmeIdA (J. L.), 1936. - Sobre um parasito de Cheiroptera, Litomosoides brasiliensis. Rev. dept. Nac. Prod. Animal, Rio-de-Janeiro, III, 133-139.

Linstow (O. F. B. von), 1879. - Helminthologische Untersuchungen. Jl. Ver. vaterl. Naturk Württemberg, XXXV, 313-342.

-, 1889. - Helminthologisches. Arch. Naturgesh. (1888), LIV, 235-246.

Rego (A. A.), 1961. - Sobre algunas especies do genero Litomosoides Chandler 1931 (Nematoda Filarioidea). Mem. Inst. O. Cruz, LIX, 1-9.

SKRJabin (K. I.), 1934. - Aprocta semenovi n. sp., nouveau Nématode de l'œil de l'engoulevent. Ann. Parasit., XII, 362-366.

Travassos (L.), 1937. - Revisao da familia Trichostrongylidae Leiper 1912. Monog. Inst. O. Cruz (1), 1-512.

Urioste (O.), 1923. - Contribucao o estudo do Trichuris. Thèse. Rio-de-Janeiro. 54 pp.

Van Thiel (P. H.), 1925. - Deux Nématodes nouveaux d'un singe hurleur de Surinam. Ann. Parasit., III, 171-180.

Vogelsang (E. G.), 1940. - Contribucion al estudio de la parasitologia animal en Venezuela (XIII). Rev. Med. Vet. y Parasit., II, 37-40.

YAmaguti (S.), 1943. - Studies on the Helminth Fauna of Japan. Part. 43. Mammalian Nematodes, IV. Jap. Journ. of Zool., X, 427-454.

—, 1961. - Systema Helminthum, III. The Nematodes of Vertebrates. New York, 2 vol.

Yorke (W.) et MAPLestone (P. A.), 1926. - The Nematode parasites of Vertebrates. 356 pp. 


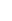

